Article

\title{
Resistive Switching Memory Devices Based on Body Fluid of Bombyx mori L.
}

\author{
Lu Wang ${ }^{1,2, *}$ and Dianzhong Wen ${ }^{1}$ \\ 1 HLJ Province Key Laboratory of Senior-Education for Electronic Engineering, Heilongjiang University, \\ Harbin 150080, China \\ 2 Research Center for Fiber Optic Sensing Technology National Local Joint Engineering, Heilongjiang \\ University, Harbin 150080, China \\ * Correspondence: wanglu@hlju.edu.cn; Tel.: +86-188-4502-5666
}

Received: 15 July 2019; Accepted: 13 August 2019; Published: 16 August 2019

\begin{abstract}
Resistive switching memory devices are strong candidates for next-generation data storage devices. Biological memristors made from renewable natural biomaterials are very promising due to their biocompatibility, biodegradability, and ecological benignity. In this study, a nonvolatile memristor was fabricated using the body fluid of Bombyx mori as the dielectric layer. The developed $\mathrm{Al} /$ Bombyx mori body fluid film/indium tin oxide (ITO) biomemristor exhibited bipolar resistive switching characteristics with a maximum on/off current ratio greater than $10^{4}$. The device showed a retention time of more than $1 \times 10^{4} \mathrm{~s}$ without any signs of deterioration, thus proving its good stability and reliability. The resistive switching behavior of the Al/Bombyx mori body fluid film/ITO biological memristor is driven by the formation and breakage of conductive filaments formed by the migration of oxygen ions. This study confirms that Bombyx mori body fluid, a $100 \%$ natural, inexpensive, and abundant material, is a potential candidate as a nonvolatile biomemristor material with broad application prospects.
\end{abstract}

Keywords: resistive switching memory devices; Bombyx mori body fluid; biomemristor; natural biomaterial

\section{Introduction}

As the integration and performance of microelectronic chips follow the rapidly increasing trend predicted by Moore's law, traditional storage technologies based on complementary metal-oxide-semiconductor (CMOS) processes are gradually approaching their physical limits, and the problem of "storage wall" in computer science is becoming increasingly serious and hindering further development. Therefore, there is a need to find new electronic device and storage technologies to enable the development of new computers with larger storage capacities and faster processing speeds. The emergence of resistive memory has given researchers hope for solving these problems. Various dielectric layer materials for resistive memory have been reported [1-7], mainly including organic molecules and polymers (metal-organic complexes [8], poly(9-vinylcarbazole) (PVK) [9]), oxides [10-12] $\left(\mathrm{TiO}_{x}\right.$ [13-15], $\mathrm{SiO}_{x}$ [16,17]), carbon-based materials [16,18,19], and perovskite-type complex oxides [20] $\left(\mathrm{SrTiO}_{3}[21,22], \mathrm{BaTiO}_{4}[23,24], \mathrm{LaMnO}_{3}\right.$ [25]). These resistive memories have the advantages of low cost, low power consumption, and multistate operation. Resistive memories have become a popular topic of research in the fields of materials science, nanoscience, physics, electronics, and information science.

With the increasing awareness of the need for environmental protection, the use of natural biological materials to develop and produce green, biocompatible, and biodegradable resistive memories has attracted the attention of an increasing number of researchers, and rapid progress has been achieved 
in this domain in recent years. As previously reported, tungsten/egg albumen/indium tin oxide (ITO)/polyethylene terephthalate devices with egg white as the dielectric layer have an on/off current ratio of approximately $5 \times 10^{2}$ and can be repeatedly cycled for 100 cycles [26]. Similarly, a bipolar resistive switching memristor with egg white as the dielectric layer has an on/off current ratio of $10^{2}-10^{4}$ and can be repeatedly cycled for 120 cycles [27]. For a ferritin-based memristor, the on/off current ratio of the device can be varied from 0 to 5 by tuning the amount of iron in the ferritin [11,28]. A memristor using $\mathrm{K}$-carrageenan ( $\mathrm{K}$-car) as the resistive switching layer has a low reset voltage $(\sim 0.05 \mathrm{~V})$, a high on/off current ratio $\left(>10^{3}\right)$, and low power consumption [29]. The fabrication of biomemory devices using composite membranes of deoxyribonucleic acid (DNA) and lead sulfide ( $\mathrm{PbS}$ ) nanoparticles has also been reported. Due to the strong electrostatic interaction between the $\mathrm{PbS}$ nanoparticles and the phosphate groups of the DNA molecules, such ITO-DNA:PbS-metal devices exhibit bistable memristive effects with high on/off current ratios of close to 4 orders of magnitude [30]. A Ag/leaves/Ti/PET device made from powdered plant leaves has a low on/off current ratio of approximately 30 and a retention time of $10^{3} \mathrm{~s}$, allowing it to function as resistive random access memory (RRAM) [31]. A Ag/cellulose fiber/Al storage device, with an active layer composed of microfibers, has also been fabricated using an electrospinning technique. This device can withstand $6 \times 10^{3}$ cycles, but its on/off current ratio is only approximately 10. The memristive effect arises due to the formation and breakage of Ag conductive filaments in the active cellulose fiber layer [32]. A fibroin protein liquid solution obtained by boiling and degumming cocoons has been used to fabricate an ITO/fibroin/Al memristor. This memristor has a low on/off current ratio of approximately 10 [33].

This paper presents an Al/Bombyx mori body fluid film/ITO biomemristor, which was fabricated using a natural biomaterial, namely, Bombyx mori body fluid, as the active layer. Experimental results showed that the device exhibited nonvolatile bipolar resistance characteristics, with a maximum on/off current ratio of greater than $10^{4}$ and a retention time of more than $1 \times 10^{4} \mathrm{~s}$, and it could withstand more than 100 write-read-erase-read cycles. The Bombyx mori body fluid used in this study can be directly used in the production of biological memristors without any purification, degumming, or other treatment processes. Compared with most other biological memristors, the presented $\mathrm{Al} /$ Bombyx mori body fluid film/ITO device exhibits higher performance. Moreover, its production process is simpler.

\section{Materials and Methods}

\subsection{Materials and Service Fabrication}

The Al/Bombyx mori body fluid film/ITO memristor was manufactured in a clean laboratory. In an ultrasonic cleaner, an ITO/glass substrate was washed successively with deionized water, acetone, and ethanol, each for $15 \mathrm{~min}$. The Bombyx mori body fluid was spin-coated onto the ITO/glass substrate (first at $500 \mathrm{rpm}$ for $5 \mathrm{~s}$ and then at $4000 \mathrm{rpm}$ for $40 \mathrm{~s}$ ), and the device was then baked at $105^{\circ} \mathrm{C}$ for $10 \mathrm{~min}$. If the drying treatment were to be carried out at a lower temperature, the device performance might be affected due to the high residual water content. Finally, an Al electrode was deposited on the Bombyx mori body fluid film via thermal evaporation to serve as the upper electrode of the biomemristor. The single Al electrode had a diameter of $400 \mu \mathrm{m}$ and a thickness of $220 \mathrm{~nm}$.

\subsection{Characterization}

The microstructure of the Bombyx mori body fluid was observed with a transmission electron microscope (TEM) (JEM-2100, JOEL, Tokyo, Japan). A scanning electron microscope (SEM) (Hitachi SU8020, Hitachi, Tokyo, Japan) was used to observe the cross section of the ITO/glass substrate coated with Bombyx mori body fluid. The Fourier transform infrared (FT-IR) (PERKIN-ELMER, Waltham, MA, USA) spectrum of the Bombyx mori body fluid and the ultraviolet-visible absorption and fluorescence emission spectra of the Bombyx mori body fluid film were also measured. Electrochemical analysis was then carried out on the Bombyx mori body fluid film using an electrochemical workstation (BAS-100B, BAS, West Lafayette, IN, USA). The electrical properties of the Al/Bombyx mori body fluid film/ITO 
memristor were tested using a semiconductor parametric tester (Keithley 4200, Keithley, Solon, ME, USA). A dynamic signal analyzer (Agilent 35670 A, Agilent, Palo Alto, CA, USA) and a current amplifier circuit were used to test the low-frequency noise of the biomemristor.

\section{Results}

Bombyx mori is an insect that undergoes complete metamorphosis. In its lifetime, it passes through four distinct morphological and functional developmental stages: egg, larva, chrysalis, and moth (adult). The egg stage corresponds to embryogenesis and the development of the larva. The larval stage mainly consists of nutrient intake and growth. The silkworm chrysalis is the stage of metamorphosis from larva to adult. The adult stage is the reproductive stage, in which mating and breeding occur. The Bombyx mori body fluid used in this experiment was obtained directly from Bombyx mori larvae. This body fluid, which is mainly composed of blood cells and plasma, accounts for 21-25\% of the body weight of the Bombyx mori.

Figure $1 \mathrm{a}$ is a photograph of a Bombyx mori, and Figure $1 \mathrm{~b}$ is a photograph of body fluid directly obtained from a Bombyx mori. The microstructure of the Bombyx mori body fluid was observed via TEM, as shown in Figure 1c, and the cross section of the body fluid/ITO/glass structure was observed via SEM, as shown in Figure 1d. In the SEM image, the upper layer is a $100 \mathrm{~nm}$ film of Bombyx mori body fluid, the middle layer is a $200 \mathrm{~nm}$ ITO film, and the lower layer is glass. Figure 1e shows the final structure of the Al/Bombyx mori body fluid film/ITO biological memristor. From top to bottom, the layers are Al (upper electrode), Bombyx mori body fluid film, ITO (lower electrode), and glass.

The chemical bonds in the Bombyx mori body fluid were analyzed via infrared spectroscopy. Figure 2a shows the FT-IR spectrum of the Bombyx mori body fluid. The peak at $1418 \mathrm{~cm}^{-1}$ was associated with the bending vibrations of carboxylate $(\mathrm{C}-\mathrm{O})$ [34]. The peak at $1635 \mathrm{~cm}^{-1}$ corresponded to the stretching vibrations of carboxyl groups $(\mathrm{C}=\mathrm{O})$ [35]. From 2340 to $3800 \mathrm{~cm}^{-1}$, wide peaks associated with the stretching vibrations of hydroxyl groups $\left(\mathrm{OH}, \sim 3445 \mathrm{~cm}^{-1}\right)$ [34] could be clearly observed.

The energy levels of the Bombyx mori body fluid were analyzed. First, an electrochemical analysis of the Bombyx mori body fluid film was performed to determine the highest occupied molecular orbital (HOMO) of the material. The cyclic voltammetry curve of the film obtained in a $0.02 \mathrm{~mol} / \mathrm{L}$ hydrochloric acid solution is shown in Figure $2 b$. As seen from this figure, the initial oxidation potential of the saturated calomel electrode relative to the vacuum level $\left(E_{O X}\right)$ was $-0.193 \mathrm{~V}$. The HOMO energy level can be obtained via the following formula [36]:

$$
E_{H O M O}=-4.74-E_{O X}
$$

Using this formula, $E_{H O M O}=-4.547 \mathrm{eV}$ could be calculated.

The ultraviolet-visible spectrum of the Bombyx mori body fluid film was measured, as shown in Figure 2c. Using this spectrum, the band gap width $\left(E_{g}\right)$ could be determined. $E_{g}$ is the difference in energy between the HOMO and the lowest unoccupied molecular orbital (LUMO). The wavelength corresponding to $E_{\mathrm{g}}$ can be calculated from the intersection of the tangent of the starting edge of the peak with the baseline in the ultraviolet-visible absorption spectrum. In this way, a $\lambda$ value of $434 \mathrm{~nm}$ was calculated. According to the relation $E g=h c / \lambda$, the band gap width of the Bombyx mori body fluid was thus calculated to be $2.857 \mathrm{eV}$. Then, the LUMO energy level could be obtained via the following formula [36]:

$$
E_{L U M O}=E_{H O M O}+E_{g}
$$

Using this formula, $E_{L U M O}=-1.690 \mathrm{eV}$ could be calculated. The energy levels of the various materials used in the Al/Bombyx mori body fluid film/ITO biomemristor are summarized in Figure $2 \mathrm{~d}$. 
(a)

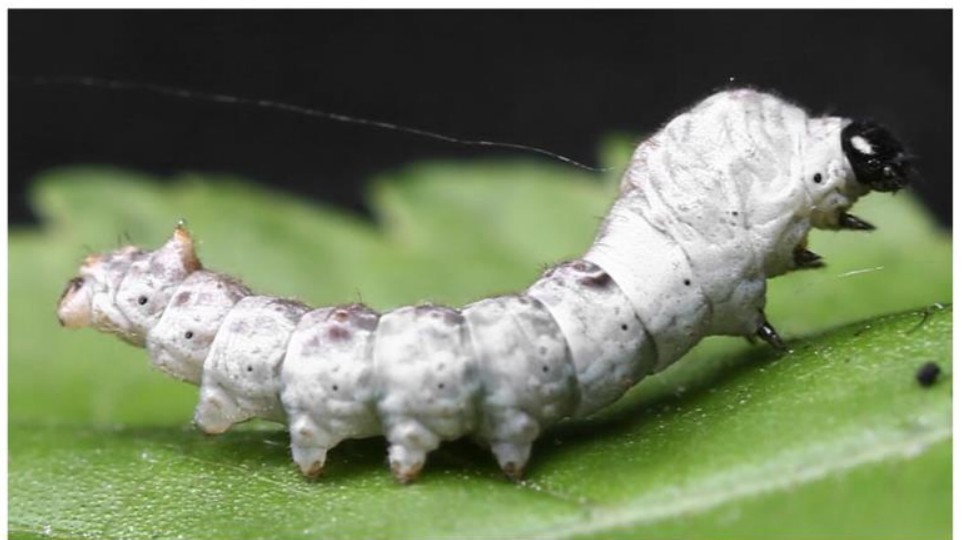

(b)

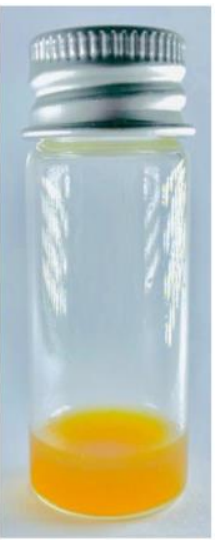

(c)

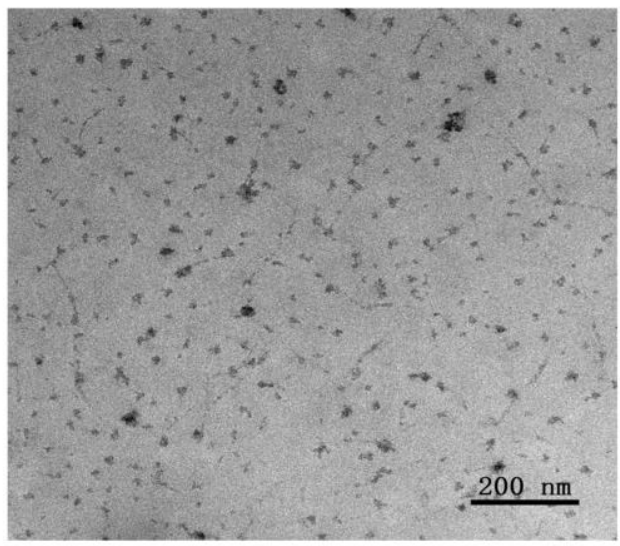

(d)
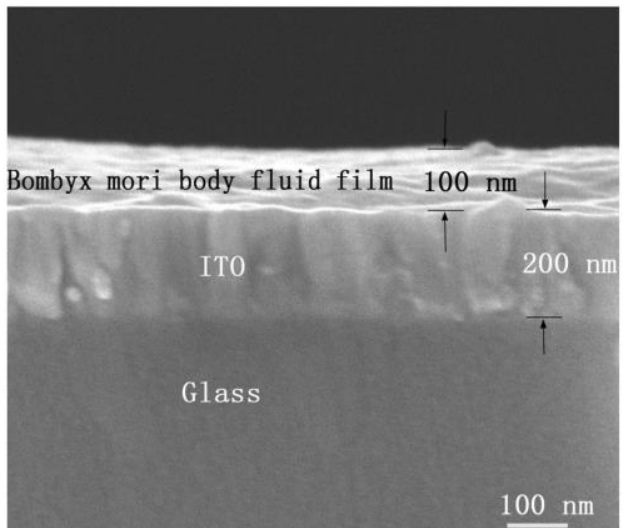

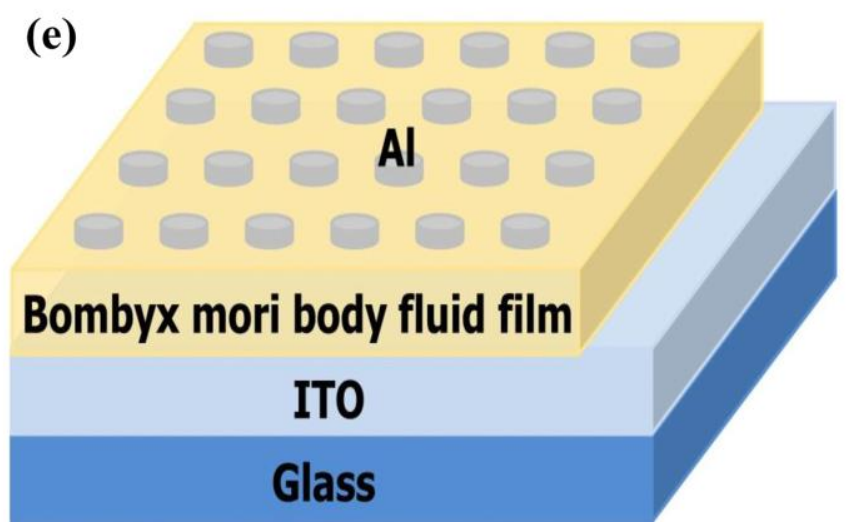

Figure 1. (a) Photograph of a Bombyx mori; (b) Photograph of Bombyx mori body fluid; (c) TEM image of Bombyx mori body fluid; (d) SEM image of the cross section of the Bombyx mori body fluid film/indium tin oxide (ITO)/glass structure; (e) Schematic diagram of the Al/Bombyx mori body fluid film/ITO biomemristor.

To further analyze the optical properties of the Bombyx mori body fluid film, its fluorescence emission spectra at excitation wavelengths of $280,300,320,340$, and $360 \mathrm{~nm}$ were measured, as shown in Figure 2e. According to the results, when the excitation wavelength was in the range of 280 to $360 \mathrm{~nm}$, the fluorescence intensity of the Bombyx mori body fluid film increased as the excitation wavelength increased. 

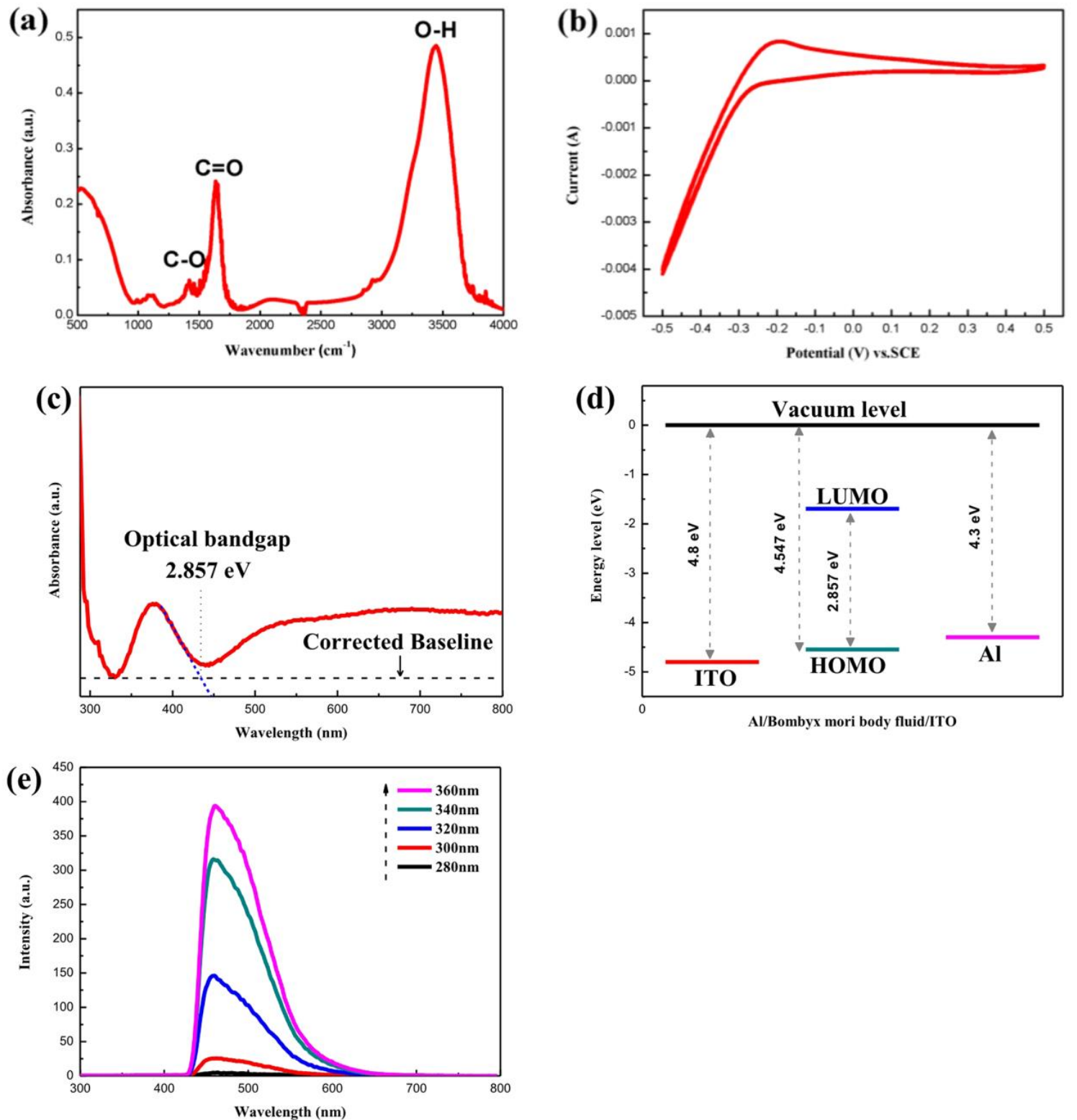

Figure 2. (a) Infrared spectrum of Bombyx mori body fluid; (b) Cyclic voltammetry curves and (c) Ultraviolet-visible absorption spectrum of the Bombyx mori body fluid film; (d) Energy level diagrams of the materials used in the Al/Bombyx mori body fluid film/ITO biomemristor; (e) Fluorescence emission spectra of the Bombyx mori body fluid film at different excitation wavelengths.

The Al/Bombyx mori body fluid film/ITO biomemristor exhibited typical bipolar resistive switching characteristics and rewritable flash characteristics. Figure 3 shows a typical SET-RESET cycle for the biomemristor. During this test, a voltage was applied to the Al electrode of the device, and the ITO electrode was grounded. The applied voltage cycle was $5 \mathrm{~V} \rightarrow 0 \mathrm{~V} \rightarrow-5 \mathrm{~V} \rightarrow 0 \mathrm{~V} \rightarrow 5 \mathrm{~V}$ with a step size of $0.05 \mathrm{~V}$, and the limiting current was set to $0.1 \mathrm{~A}$. When an applied voltage $(5 \mathrm{~V} \rightarrow 0 \mathrm{~V} \rightarrow-5 \mathrm{~V})$ was applied to the $\mathrm{Al}$ electrode, the device was initially in a high-resistance state (HRS), that is, the "off state". As the bias voltage decreased, the current also initially showed a steady decrease. When the applied voltage reached $-0.85 \mathrm{~V}$ (defined as the set voltage, $V_{S E T}$ ), the current suddenly increased. The current of the device suddenly changed from $4.87 \times 10^{-6} \mathrm{~A}$ to $1.15 \times 10^{-2} \mathrm{~A}$, that is, the device suddenly changed from an HRS to a low-resistance state (LRS), meaning that the device switched to the "on state". This process is called the SET process. After the device was set, the current varied with the voltage, but the device remained in the LRS. Then, when a voltage cycle of $-5 \mathrm{~V} \rightarrow 0 \mathrm{~V} \rightarrow 5 \mathrm{~V}$ 
was applied to the device, when the voltage reached $3.05 \mathrm{~V}$ (defined as the reset voltage, $V_{\text {RESET }}$ ), the current suddenly decreased. At this time, the current in the biomemristor suddenly changed from $7.18 \times 10^{-2} \mathrm{~A}$ to $2.01 \times 10^{-5} \mathrm{~A}$, and the device changed from the LRS to the HRS. This process is called the RESET process.

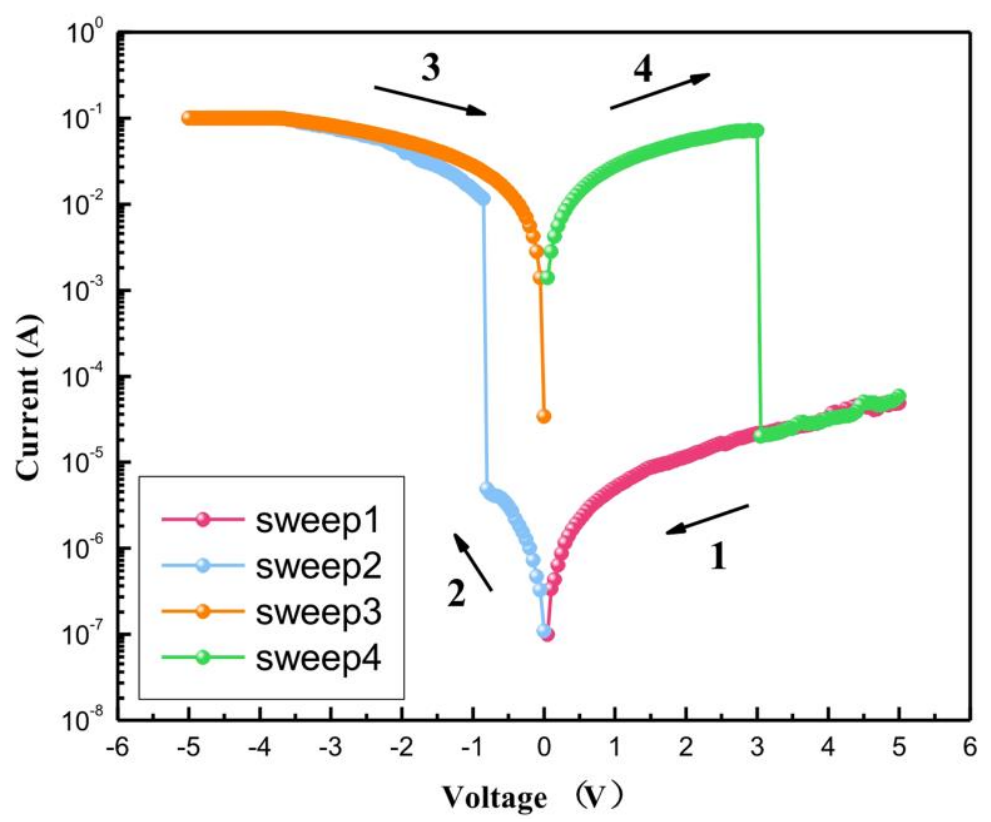

Figure 3. Current-voltage (I-V) characteristics of Al/Bombyx mori body fluid film/ITO biomemristor.

Figure 4 shows the variation in the on/off current ratio of the Al/Bombyx mori body fluid film/ITO memristor with the applied voltage. When the voltage was varied from -0.80 to $3.00 \mathrm{~V}$, the on/off current ratio was between $3.34 \times 10^{3}$ and $1.41 \times 10^{4}$. When $\mathrm{V}=0.05$, the biomemristor had a maximum on/off current ratio of $1.41 \times 10^{4}$. Compared with other biological memristors, the $\mathrm{Al} /$ Bombyx mori body fluid film/ITO memristor had a relatively high on/off current ratio, indicating that this device has good application prospects as a type of nonvolatile memory.

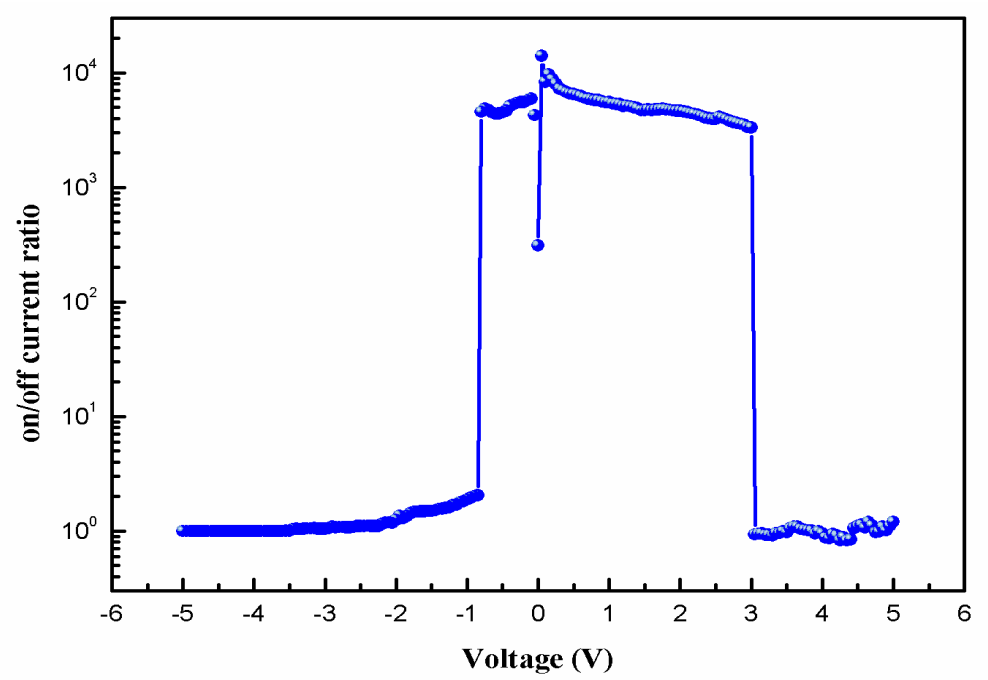

Figure 4. Variation in the on/off current ratio of Al/Bombyx mori body fluid film/ITO biomemristor with the applied voltage.

Figure $5 \mathrm{a}$ is the superposition I-V curves of two biomemristor cells from the same glass substrate measured 15 times for each cell. It can be seen from this figure that the values of $V_{\text {SET }}$ and $V_{\text {RESET }}$ 
changed with cyclic switching. The average of the $V_{S E T}$ for the biomemristor was $-0.89 \mathrm{~V}$, whereas that of the $V_{R E S E T}$ was $3.34 \mathrm{~V}$. Figure $5 \mathrm{~b}$ shows the cumulative probabilities of the resistances of HRS $\left(R_{H R S}\right)$ and LRS $\left(R_{L R S}\right) . R_{L R S}$ demonstrated narrow distributions. Figure $5 \mathrm{c}$ shows the $V_{S E T}$ and $V_{R E S E T}$ distributions of the biomemristors, which were fitted using Gaussian function. Differences between the $V_{S E T}$ and $V_{R E S E T}$ of the biomemristors were sufficiently large to ensure that the HRS and LRS of the devices were distinguishable.
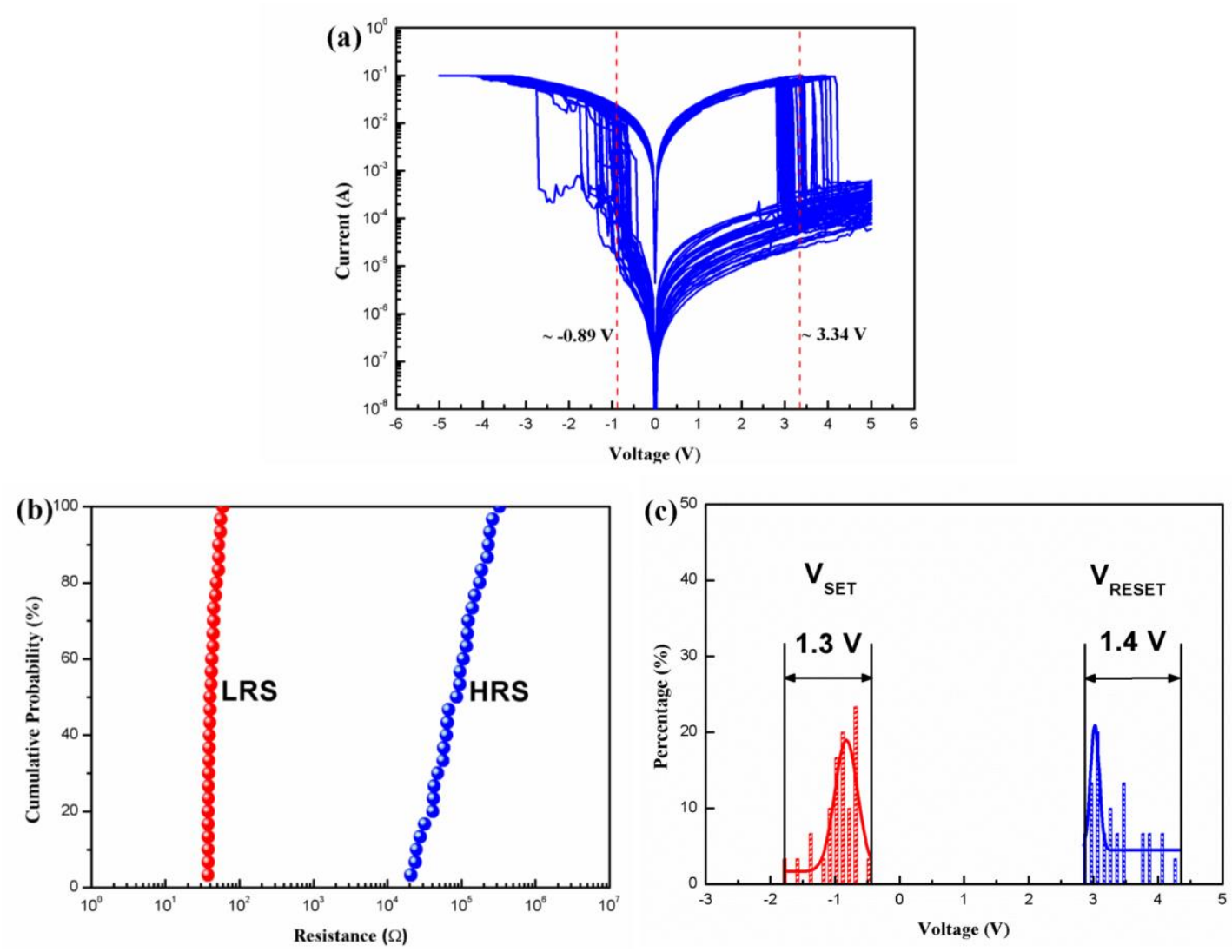

Figure 5. (a) Superposition of I-V curves for two biomemristor cells from the same glass substrate measured 15 times for each cell; (b) Cumulative probabilities of the resistance of high-resistance state $\left(R_{H R S}\right)$ and resistance of low-resistance state $\left(R_{L R S}\right)$ of the devices; (c) $V_{S E T}$ and $V_{R E S E T}$ distributions of the biomemristors.

Figure 6a shows the retention properties of the Al/Bombyx mori body fluid film/ITO biomemristor. At $1 \mathrm{~V}$, the HRS and LRS of the device remained basically unchanged for $10^{4} \mathrm{~s}$, which means that the device could be stably maintained in either the HRS or the LRS, demonstrating the nonvolatility of the memristor. Figure $6 \mathrm{~b}$ shows the endurance properties of the biomemristor. The device could be written to and erased by applying pulse signals of $4.5 \mathrm{~V} / 100 \mathrm{~ms}$ and $-3.5 \mathrm{~V} / 100 \mathrm{~ms}$, respectively. The current of the device was measured at $1 \mathrm{~V}$ after each operation. The biomemristor showed rewritable performance over 100 cycles. 

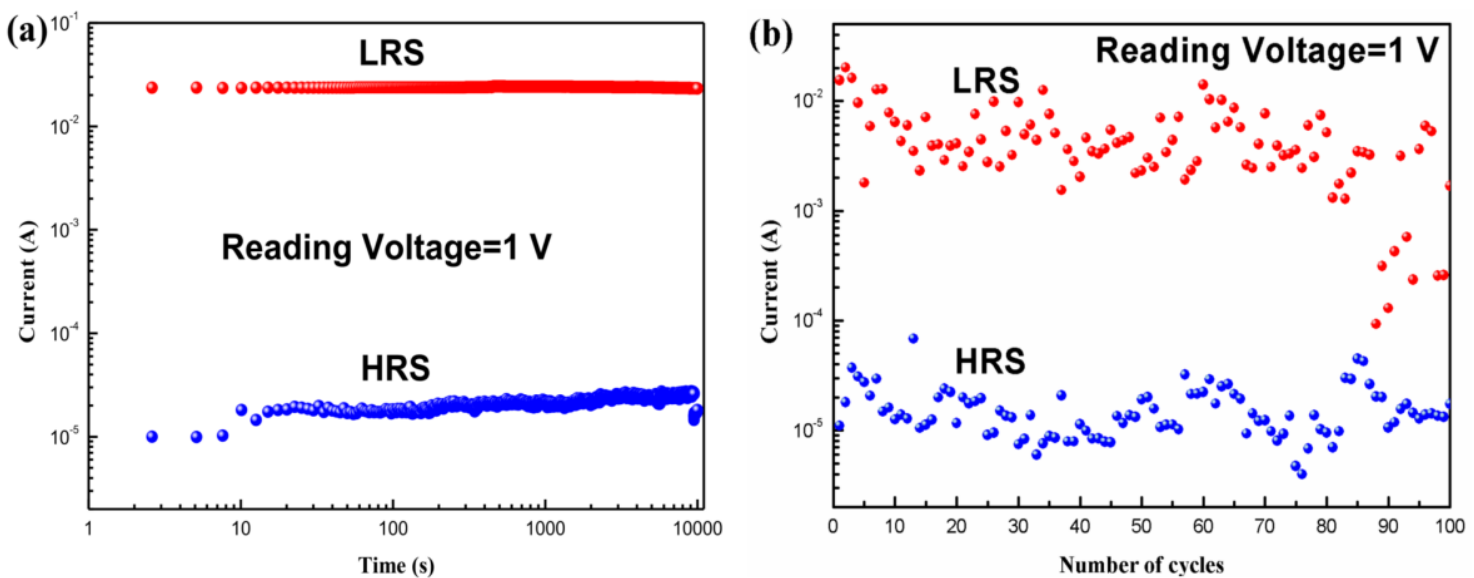

Figure 6. (a) Retention properties; (b) Endurance properties of Al/Bombyx mori body fluid film/ITO biomemristor.

To study the current transfer mechanism of the biomemristor, we replotted the I-V characteristic curve of the device in double natural logarithmic coordinates, as shown in Figure 7. As can be seen, the slope of the current-voltage characteristic curve was approximately 1 in both the LRS and the HRS, that is, the current and voltage were linear, $\mathrm{I} \propto \mathrm{V}$, indicating that the conduction current followed Ohm's law.

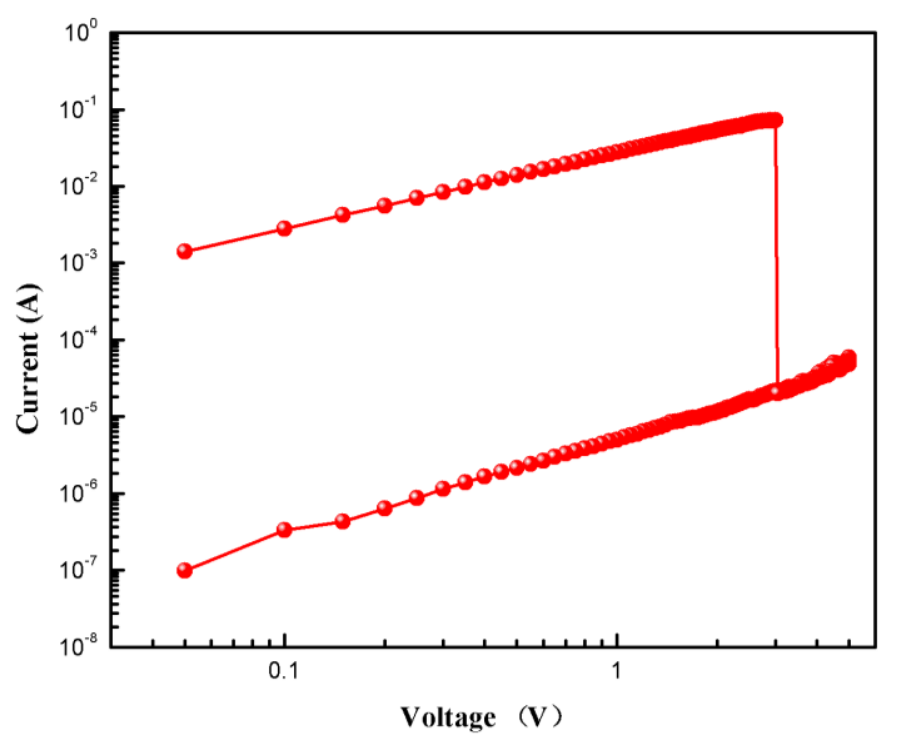

Figure 7. I-V characteristics of Al/Bombyx mori body fluid film/ITO biomemristor in double natural logarithmic coordinates.

To study the resistive switching mechanism of the Al/Bombyx mori body fluid film/ITO biomemristor, a low-frequency noise power spectral density experiment was carried out. Figure 8 shows the current noise power spectral density of the device for applied voltages of 0.001 and $0.008 \mathrm{~V}$, respectively. It can be seen from this figure that the power spectral density of the current noise in the LRS was higher than that in the HRS. In addition, the power spectral density of the current noise was inversely proportional to the frequency, that is, in the region of the noise spectrum below $1600 \mathrm{~Hz}$, the noise of the device increased sharply as the frequency decreased. The relationship between the noise and the frequency was $1 / f$. Under different applied voltages, the slope of the power spectral density curve of the current noise was approximately 1 in both the HRS and the LRS, indicating that the noise of the Al/Bombyx mori body fluid film/ITO biomemristor was 1/f noise. It can be inferred that, in this biomemristor, 
the formation and breakage of conductive filaments caused the device to exhibit resistive switching behavior [37].
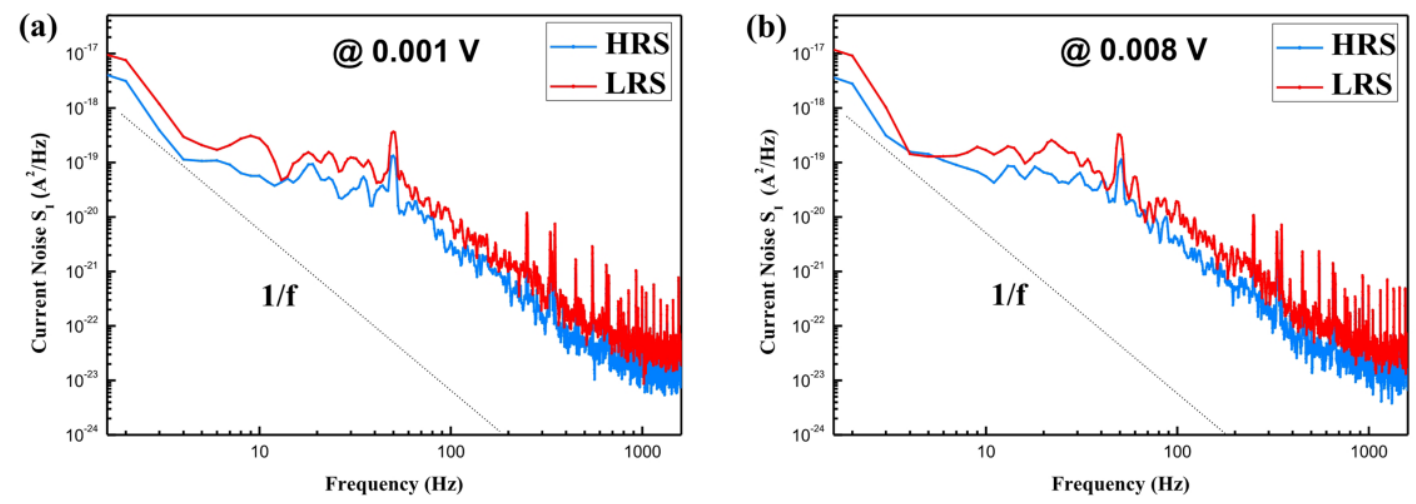

Figure 8. Current noise power spectral density of the Al/Bombyx mori body fluid film/ITO biomemristor in the LRS and the HRS under applied voltages of (a) $0.001 \mathrm{~V}$ and (b) $0.008 \mathrm{~V}$.

Bombyx mori body fluid is a circulating liquid that bathes all tissues and organs in the body cavity. This fluid contains dry matter in addition to a large amount of water. The main components of the dry matter are proteins, amino acids, other nonprotein nitrogen compounds, trehalose, phosphate, and citrate as well as metal ions, such as sodium, potassium, calcium, and magnesium. It can be seen from the infrared spectrum of the Bombyx mori body fluid in Figure $2 \mathrm{a}$ that this fluid contains carboxylate $(\mathrm{C}-\mathrm{O})$, carboxyl $(\mathrm{C}=\mathrm{O})$, and hydroxyl $(\mathrm{OH})$ functional groups. Based on the above analysis, we conclude that the resistive switching properties of this biomemristor mainly originate from the migration of oxygen ions. Accordingly, we have drawn a schematic diagram of the resistive switching mechanism of the developed Al/Bombyx mori body fluid film/ITO biomemristor, as shown in Figure 9. When no bias voltage is applied, most oxygen ions in the Bombyx mori body fluid film are distributed, as shown in Figure 9a. When a negative voltage is applied to the upper electrode (Al electrode), negatively charged oxygen ions in the Bombyx mori body fluid film gradually accumulate on the side closer to the lower electrode (ITO electrode), and they gradually diffuse toward the upper electrode, as shown in Figure $9 \mathrm{~b}$. When a sufficiently large negative voltage $V_{S E T}$ is applied, a strong conductive path (which we call a conductive filament) is finally formed, which penetrates the Bombyx mori body fluid layer and connects the upper and lower electrodes, as shown in Figure 9c. At this time, the HRS is converted into an LRS, and the device completes the "write" operation. In contrast, when a positive voltage is applied to the upper electrode (Al electrode), the electric charge forming the conductive filament is gradually dissipated. When a sufficiently large positive voltage $\left(V_{R E S E T}\right)$ is applied, the conductive filament breaks, and the device transitions from the LRS to the HRS, completing the "erase" operation, as shown in Figure 9d.

Therefore, under the action of an electric field, the migration of oxygen ions leads to the formation and breakage of conductive filaments, serving as the resistive switching mechanism of the developed Al/Bombyx mori body fluid film/ITO biomemristor. 
(a)

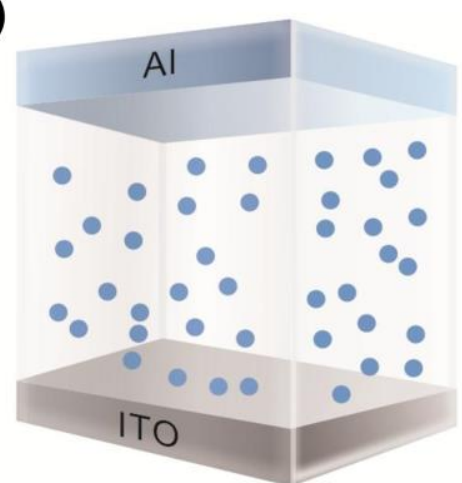

(c)

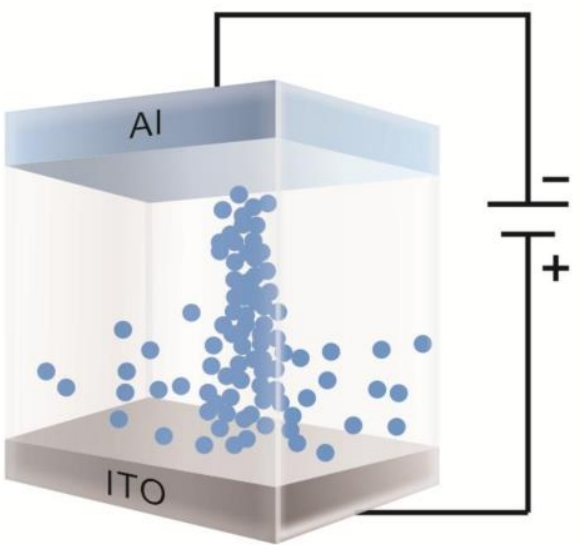

(b)

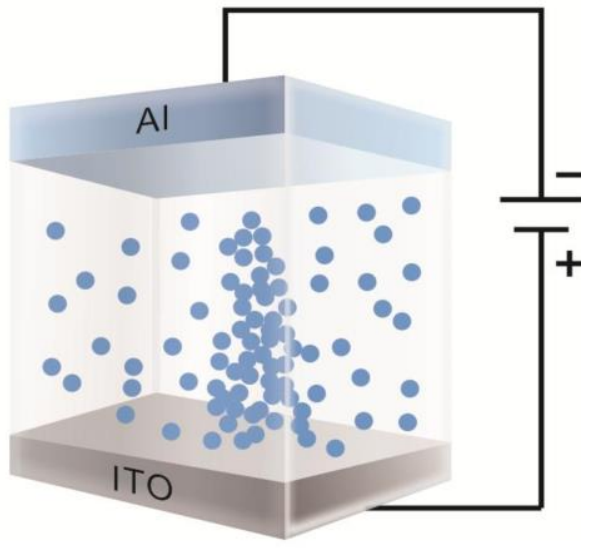

(d)

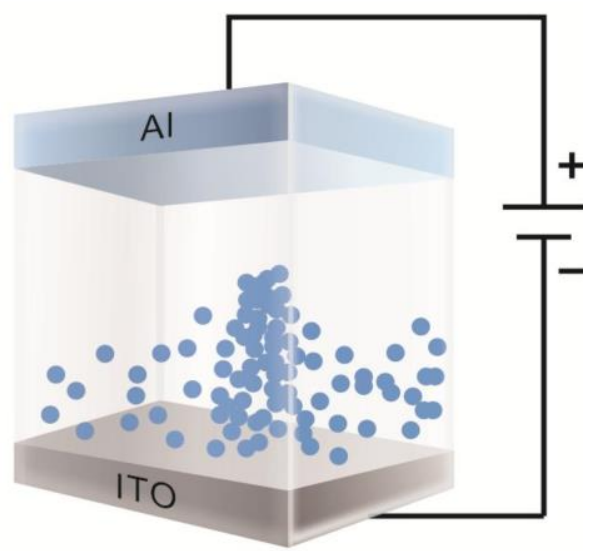

Figure 9. Schematic diagram of the resistive switching mechanism of the $\mathrm{Al} /$ Bombyx mori body fluid film/ITO biomemristor: (a) OFF; (b) SET; (c) ON; (d) RESET.

\section{Conclusions}

In conclusion, we successfully developed an Al/Bombyx mori body fluid film/ITO biomemristor, which exhibited nonvolatile resistive switching characteristics with a maximum on/off current ratio greater than $10^{4}$. The device could store information for more than $1 \times 10^{4} \mathrm{~s}$ without any deterioration, thus showing its high stability and reliability.

The Al/Bombyx mori body fluid film/ITO biomemristor, in which the active layer consists of Bombyx mori body fluid, has the advantages of biodegradability, biocompatibility, and environmental friendliness and has broad application prospects in the fields of bioelectronics, information storage, and artificial neural networks. This study is the first to demonstrate that Bombyx mori body fluid is a promising natural biomaterial for use in nonvolatile resistive memory, perhaps in future implantable and biocompatible nanoelectronics.

Author Contributions: L.W. designed this work, performed the experiments, and wrote the manuscript. L.W. and D.W. analyzed and discussed the experimental results.

Funding: This work was supported by the National Natural Science Foundation of China (Grant No. 61801174), the Basic Research Project funded by the Basic Research Business Funding for Provincial Universities in Heilongjiang Province (Grant No. KJCX201808), the Science and Technology Innovative Research Team in Higher Educational Institutions of Heilongjiang Province (No. 2012TD007), and the Electronic Engineering Provincial Key Laboratory.

Conflicts of Interest: The authors declare no conflict of interest. 


\section{References}

1. Hosseini, N.R.; Lee, J.-S. Resistive switching memory based on bioinspired natural solid polymer electrolytes. ACS Nano 2014, 9, 419-426. [CrossRef] [PubMed]

2. Hosseini, N.R.; Lee, J.-S. Biocompatible and flexible chitosan-based resistive switching memory with magnesium electrodes. Adv. Funct. Mater. 2015, 25, 5586-5592. [CrossRef]

3. Hosseini, N.R.; Lee, J.-S. Controlling the resistive switching behavior in starch-based flexible biomemristors. ACS Appl. Mater. Interfaces 2016, 8, 7326-7332. [CrossRef] [PubMed]

4. Dutta, M.; Maikap, S.; Qiu, J.T. Controlling conductive filament and tributyrin sensing using an optimized porous iridium interfacial layer in $\mathrm{Cu} / \mathrm{Ir} / \mathrm{TiNxOy} / \mathrm{TiN}$. Adv. Electron. Mater. 2019, 5, 1800288.

5. Qiu, J.T.; Samanta, S.; Dutta, M.; Ginnaram, S.; Maikap, S. Controlling resistive switching by using an optimized $\mathrm{MoS}_{2}$ interfacial layer and the role of top electrodes on ascorbic acid sensing in $\mathrm{TaO}_{x}$-based RRAM. Langmuir 2019, 35, 3897-3906. [CrossRef] [PubMed]

6. Jo, S.; Sun, W.; Kim, B.; Kim, S.; Park, J.; Shin, H. Memristor neural network training with clock synchronous neuromorphic system. Micromachines 2019, 10, 384. [CrossRef] [PubMed]

7. Pham, K.V.; Nguyen, T.V.; Min, K.-S. Partial-gated memristor crossbar for fast and power-efficient defect-tolerant training. Micromachines 2019, 10, 245. [CrossRef]

8. Goswami, S.; Matula, A.J.; Rath, S.P.; Hedström, S.; Saha, S.; Annamalai, M.; Sengupta, D.; Patra, A.; Ghosh, S.; Jani, H. Robust resistive memory devices using solution-processable metal-coordinated azo aromatics. Nat. Mater. 2017, 16, 1216. [CrossRef]

9. Zhang, X.; Li, J.; Yang, W.; Leng, B.; Niu, P.; Jiang, X.; Liu, B. High-performance flexible UV photodetectors based on AZO/ZnO/PVK/PEDOT: PSS heterostructures integrated on human hair. ACS Appl. Mater. Interfaces 2019, 11, 24459-24467. [CrossRef]

10. Kumar, D.; Aluguri, R.; Chand, U.; Tseng, T. Metal oxide resistive switching memory: Materials, properties and switching mechanisms. Ceram. Int. 2017, 43, S547-S556. [CrossRef]

11. Wang, H.; Meng, F.; Zhu, B.; Leow, W.R.; Liu, Y.; Chen, X. Resistive switching memory devices based on proteins. Adv. Mater. 2015, 27, 7670-7676. [CrossRef] [PubMed]

12. Yoon, J.H.; Zhang, J.; Ren, X.; Wang, Z.; Wu, H.; Li, Z.; Barnell, M.; Wu, Q.; Lauhon, L.J.; Xia, Q. Truly electroforming-free and low-energy memristors with preconditioned conductive tunneling paths. Adv. Funct. Mater. 2017, 27, 1702010. [CrossRef]

13. Banerjee, W.; Liu, Q.; Lv, H.; Long, S.; Liu, M. Electronic imitation of behavioral and psychological synaptic activities using $\mathrm{TiO}_{x} / \mathrm{Al}_{2} \mathrm{O}_{3}$-based memristor devices. Nanoscale 2017, 9, 14442-14450. [CrossRef] [PubMed]

14. Srivastava, S.; Thomas, J.P.; Heinig, N.F.; Leung, K. High-performance single-active-layer memristor based on an ultrananocrystalline oxygen-deficient $\mathrm{TiO}_{\mathrm{x}}$ film. ACS Appl. Mater. Interfaces 2017, 9, 36989-36996. [CrossRef] [PubMed]

15. Yu, Y.; Wang, C.; Jiang, C.; Abrahams, I.; Du, Z.; Zhang, Q.; Sun, J.; Huang, X. Resistive switching behavior in memristors with $\mathrm{TiO}_{2}$ nanorod arrays of different dimensions. Appl. Surf. Sci. 2019, 485, 222-229. [CrossRef]

16. He, C.; Li, J.; Wu, X.; Chen, P.; Zhao, J.; Yin, K.; Cheng, M.; Yang, W.; Xie, G.; Wang, D. Tunable electroluminescence in planar graphene/ $\mathrm{SiO}_{2}$ memristors. Adv. Mater. 2013, 25, 5593-5598. [CrossRef] [PubMed]

17. Mehonic, A.; Shluger, A.L.; Gao, D.; Valov, I.; Miranda, E.; Ielmini, D.; Bricalli, A.; Ambrosi, E.; Li, C.; Yang, J.J. Silicon oxide $\left(\mathrm{SiO}_{\mathrm{x}}\right)$ : A promising material for resistance switching? Adv. Mater. 2018, 30, 1801187. [CrossRef] [PubMed]

18. Pi, S.; Li, C.; Jiang, H.; Xia, W.; Xin, H.; Yang, J.J.; Xia, Q. Memristor crossbar arrays with 6-nm half-pitch and 2-nm critical dimension. Nat. Nanotechnol. 2019, 14, 35. [CrossRef]

19. Sysoev, V.; Arkhipov, V.; Okotrub, A.; Pershin, Y. Chemical sensors are hybrid-input memristors. Appl. Surf. Sci. 2018, 436, 1018-1021. [CrossRef]

20. Upadhyay, N.K.; Jiang, H.; Wang, Z.; Asapu, S.; Xia, Q.; Joshua Yang, J. Emerging memory devices for neuromorphic computing. Adv. Mater. Technol. 2019, 4, 1800589. [CrossRef]

21. Jang, J.T.; Ko, D.; Ahn, G.; Yu, H.R.; Jung, H.; Kim, Y.S.; Yoon, C.; Lee, S.; Park, B.H.; Choi, S.-J. Effect of oxygen content of the $\mathrm{LaAlO}_{3}$ layer on the synaptic behavior of $\mathrm{Pt} / \mathrm{LaAlO}_{3} / \mathrm{Nb}$-doped $\mathrm{SrTiO}_{3}$ memristors for neuromorphic applications. Solid-State Electron. 2018, 140, 139-143. [CrossRef] 
22. Nili, H.; Walia, S.; Kandjani, A.E.; Ramanathan, R.; Gutruf, P.; Ahmed, T.; Balendhran, S.; Bansal, V.; Strukov, D.B.; Kavehei, O. Donor-induced performance tuning of amorphous $\mathrm{SrTiO}_{3}$ memristive nanodevices: Multistate resistive switching and mechanical tunability. Adv. Funct. Mater. 2015, 25, 3172-3182. [CrossRef]

23. Huang, W.; Fang, Y.-W.; Yin, Y.; Tian, B.; Zhao, W.; Hou, C.; Ma, C.; Li, Q.; Tsymbal, E.Y.; Duan, C.-G. Solid-state synapse based on magnetoelectrically coupled memristor. ACS Appl. Mater. Interfaces 2018, 10, 5649-5656. [CrossRef]

24. Wei, L.; Yuan, Y.; Wang, J.; Tu, H.; Gao, Y.; You, B.; Du, J. Bipolar resistive switching with negative differential resistance effect in a $\mathrm{Cu} / \mathrm{BaTiO}_{3} / \mathrm{Ag}$ device. Phys. Chem. Chem. Phys. 2017, 19, 11864-11868. [CrossRef]

25. Xu, Z.T.; Jin, K.J.; Gu, L.; Jin, Y.L.; Ge, C.; Wang, C.; Guo, H.Z.; Lu, H.B.; Zhao, R.Q.; Yang, G.Z. Evidence for a crucial role played by oxygen vacancies in $\mathrm{LaMnO}_{3}$ resistive switching memories. Small 2012, 8, 1279-1284. [CrossRef] [PubMed]

26. Yan, X.; Li, X.; Zhou, Z.; Zhao, J.; Wang, H.; Wang, J.; Zhang, L.; Ren, D.; Zhang, X.; Chen, J. A flexible transparent organic artificial synapse based on tungsten/egg albumen/indium tin oxide/polyethylene terephthalate memristor. ACS Appl. Mater. Interfaces 2019, 11, 18654-18661. [CrossRef] [PubMed]

27. He, X.; Zhang, J.; Wang, W.; Xuan, W.; Wang, X.; Zhang, Q.; Smith, C.G.; Luo, J. Transient resistive switching devices made from egg albumen dielectrics and dissolvable electrodes. ACS Appl. Mater. Interfaces 2016, 8, 10954-10960. [CrossRef] [PubMed]

28. Meng, F.; Sana, B.; Li, Y.; Liu, Y.; Lim, S.; Chen, X. Bioengineered tunable memristor based on protein nanocage. Small 2014, 10, 277-283. [CrossRef]

29. Kim, M.-K.; Lee, J.-S. Ultralow power consumption flexible biomemristors. ACS Appl. Mater. Interfaces 2018, 10, 10280-10286. [CrossRef]

30. Murgunde, B.K.; Rabinal, M.K.; Kalasad, M.N. Biologically active nanocomposite of DNA-PbS nanoparticles: A new material for non-volatile memory devices. Appl. Surf. Sci. 2018, 427, 344-353. [CrossRef]

31. Sun, B.; Zhu, S.; Mao, S.; Zheng, P.; Xia, Y.; Yang, F.; Lei, M.; Zhao, Y. From dead leaves to sustainable organic resistive switching memory. J. Colloid Interface Sci. 2018, 513, 774-778. [CrossRef] [PubMed]

32. Rananavare, A.P.; Kadam, S.J.; Prabhu, S.V.; Chavan, S.S.; Anbhule, P.V.; Dongale, T.D. Organic non-volatile memory device based on cellulose fibers. Mater. Lett. 2018, 232, 99-102. [CrossRef]

33. Hota, M.K.; Bera, M.K.; Kundu, B.; Kundu, S.C.; Maiti, C.K. A natural silk fibroin protein-based transparent bio-memristor. Adv. Funct. Mater. 2012, 22, 4493-4499. [CrossRef]

34. Sheng, G.-P.; Yu, H.-Q.; Yu, Z. Extraction of extracellular polymeric substances from the photosynthetic bacterium rhodopseudomonas acidophila. Appl. Microbiol. Biotechnol. 2005, 67, 125-130. [CrossRef] [PubMed]

35. Moreno-Castilla, C.; Lopez-Ramon, M.; Carrasco-Marın, F. Changes in surface chemistry of activated carbons by wet oxidation. Carbon 2000, 38, 1995-2001. [CrossRef]

36. Cheng, F.; He, C.; Ren, M.; Yao, L.; Tang, N. Preparation, photophysical, and electrochemical properties of three trinuclear $\mathrm{Ru}(\mathrm{II})$ complexes: Bridging ligands composed of 2,2'-bipyridine and 4,5-diazafluorene fragments. Inorg. Chem. Commun. 2014, 43, 10-14. [CrossRef]

37. Chen, Y.-C.; Yu, H.-C.; Huang, C.-Y.; Chung, W.-L.; Wu, S.-L.; Su, Y.-K. Nonvolatile bio-memristor fabricated with egg albumen film. Sci. Rep. 2015, 5, 10022. [CrossRef] [PubMed]

(C) 2019 by the authors. Licensee MDPI, Basel, Switzerland. This article is an open access article distributed under the terms and conditions of the Creative Commons Attribution (CC BY) license (http://creativecommons.org/licenses/by/4.0/). 